\title{
LA CULTURE MATÉRIELLE DES HONGROIS EN ANALYSE. DÉCOR ET MISE-EN-SCÈNE DANS LES SÉRIES TÉLÉVISÉES HONGROISES CONTEMPORAINES
}

\author{
ANNA KESZEG \\ University of Debrecen \\ akeszega@gmail.com
}

\begin{abstract}
Au cours de la dernière décennie, la Hongrie est devenue plus visible sur le marché international de la série télévisée : les productions de la chaine HBO et les productions, plus récentes, de RTL Klub contribuent à l'apparition d'une image nouvelle pour la région. L'objectif du présent article est d'analyser le choix et la décoration des lieux d'habitation dans les séries télévisées hongroises. Dans ce but, on présente d'abord les modalités du marché domestique de la production télévisée. Or l'évolution de ce marché ne peut pas être comprise sans aborder dans un premier temps l'histoire des séries dites complexes et le rôle joué par HBO dans leur développement. Les séries analysées, qui appartiennent au genre dramatique (Terápia, Aranyélet, Alvilág), font parti de ce nouveau format. La seconde partie de l'article présente les schémas théoriques d'analyse du décor cinématographique proposés par Charles et Mirella Jona Affron et par Charles Tashiro. En se basant sur les catégories respectives de ces auteurs, l'article montre que les séries hongroises adoptent de plus en plus un modèle caractérisé par une grande attention au design et où les signes de la culture matérielle jouent un rôle essentiel dans le projet de décrire les conflits d'ordre culturel, sociaux et politiques inhérents à la société hongroise contemporaine.
\end{abstract}

Mots-clés : séries télévisées, direction artistique, design, culture matérielle

Au cours de la dernière décennie, la Hongrie est devenue plus visible sur le marché international de la série télévisée : des productions réalisées par la chaîne HBO ou de nouvelles productions en cours de réalisation par RTL Klub contribuent à la naissance d'une nouvelle image de la région. L'ambition de notre article est d'analyser l'image du pays et de la culture hongroise telle qu'elle apparaît dans ces productions, notamment comment la culture matérielle contemporaine hongroise est adaptée à l'écran dans les séries de nouvelle génération. L'analyse de contenus fictifs en tant que signes d'une culture matérielle réelle nous semble justifiée par le fait même que les contenus sont destinés à produire des effets de réel. Leur interprétation en ce sens entre donc dans la logique même 
de leur production. Les séries que nous allons prendre en compte appartiennent au modèle des séries dites de nouvelle génération, sur lequel nous reviendrons plus tard : il s'agit de la série Terápia (En analyse, HBO Europe, 2012-2017), de Aranyélet (Golden life, HBO Europe, 2015-2018) et de la série Alvilág (Basfond, RTL Klub, 2019).

\section{La télévision complexe et les séries de nouvelle génération}

Au cours des cinq dernières décennies, plusieurs micro-révolutions ont eu lieu dans l'industrie télévisuelle : mentionnons la création de HBO aux États-Unis dans les années 1970 et l'introduction du concept de qualité dans le divertissement télévisuel, puis, dans les années 1980-1990, l'apparition des séries dites complexes (Mittell, 2015), et enfin la révolution digitale des années 2000 et la vague des séries de nouvelle génération accessibles en flux continu, capables d'attirer un public de plus en plus averti devant le petit écran. L'expression elle-même de "petit écran" a changé de sens : avec les services de flux continu, l'écran peut avoir les dimensions que l'on désire ou que l'on se peut se permettre. Dans le numéro spécial de Manières de voir (supplément du Monde Diplomatique) consacré aux séries télévisées, en été 2017, Dominique Pinsolle et Arnaud Rindel commencent un article consacré à $\mathrm{HBO}$ et au concept de "série de qualité" en affirmant que « les séries ont envahie la presse, font l'objet de colloques universitaires et d'ouvrages soulignant leur profondeur philosophique. » (Pinsolle - Rindel, 2017, 32.) Ils constatent que les séries ont changé de statut : de curiosités dérisoires destinées à une consommation populaire, elles sont devenues des objets de consommation liés au prestige, dignes même de l'intérêt académique.

Ce qu'on peut appeler le troisième âge d'or de la télévision se caractérise avant tout par l'introduction de la notion de qualité au sein de la production télévisuelle. Notons que le slogan de $\mathrm{HBO}$, chaîne considérée comme inventeur de ce nouveau format, entre 1997 et 2006, était It's not TV. It's HBO. (Ce n'est pas de la télévision, c'est $H B O$.) Avec ce slogan, HBO a lancé le concept de « programmation distinctive », c'est-à-dire que la chaîne promettait des œuvres d'art indépendantes de la logique commerciale propre au secteur de la télévision. De plus, le renoncement aux interruptions publicitaires qui fragmentaient les contenus filmiques a contribué à renforcer la sensation d'indépendance commerciale. Une autre conséquence de la stratégie de HBO fut l'élaboration d'un style narratif et filmique avant-gardiste qui s'accordait bien avec l'intérêt pour le cinéma d'auteur du public-cible de HBO. Les séries cultes de David Simon, The Wire (2002-2006), Treme (2010-2013), ou Show me a Hero (2015) ont fonctionné de 
la même manière que les films-cultes, reconnus pour offrir un univers filmique aisément reconnaissable car il repose sur la poétique particulière d'un réalisateur. Au demeurant, ces séries produisent un effet de réel qui permet de saisir, à travers la construction d'univers fictifs, certains processus politiques, sociaux et économiques, d'où l'intérêt professé par le monde académique à leur égard. Grâce à la série The Wire, on peut se faire, par exemple, une image assez vraisemblable de la criminalité à Baltimore : le récit est écrit selon plusieurs perspectives et l'ambition réaliste, qui se réclame du journalisme d'investigation, caractérisent cette série devenue classique dans l'univers esthétique de HBO. Il faut également remarquer la rapidité de réaction des créateurs : la série Treme, par exemple, qui documente le processus de reconstruction de la ville de Nouvelle-Orléans après les ravages de l'ouragan Katrina, en 2005, fut diffusée dans un délais de seulement cinq ans après la catastrophe. Ces productions sont caractérisées par un concept fondamental dans l'esthétique de la chaîne, à savoir un sens du drame qui est devenu l'image de marque de HBO. Les récits de crimes et les thrilleurs occupent le devant de la scène. Quant aux productions comiques, elles s'intègrent au modèle hybride de la comédie dramatique (dramedy) où l'élément comique joue un rôle secondaire dans le cadre de la construction dramatique de la narration.

D'ailleurs, cette évolution n'est pas en contradiction avec la pratique antérieure de l'industrie du film : le storytelling, en particulier l'invention de narrations complexes produisant un effet de réel, a toujours été un composant essentiel. Cette idée apparaît dans le livre de Michael Wolff, qui a enquêté sur le succès de la télévision en tant qu'ancien média plongé dans l'univers des nouveaux médias (Wolff 2015). Wolff affirme qu'au moment où Internet a permis de réinventer le format télévisuel ou de le concurrencer, la télévision se trouvait déjà dans un accord si étroit avec les attentes du nouveau public de milléniaux, que ce n'est pas Internet qui a provoqué une révolution dans le domaine télévisuel, c'est plutôt l'inverse qui s'est produit : la logique télévisuelle s'est elle-même imposée aux marchés du flux continu (Wolff 2015 : 113-114). Lors de son entrées le marché, Netflix, qui en est devenu l'acteur le plus important, a été contraint de chercher sa place parmi les chaînes de télévision classiques et a dû adopter leur type de stratégie marketing. En ce sens, la révolution réelle reste celle, plus ancienne, produite par $\mathrm{HBO}$, même si dernièrement $\mathrm{HBO}$ a été pris de vitesse sur certains marchés par d'autres acteurs comme AMC ou Showtime, etc.

Le modèle HBO se caractérise par un système de production innovateur. Les problèmes globaux, analysés à travers le récit de la vie des personnages, sont toujours fortement ancrés dans les événements des sociétés locales. Ce modèle thématique est en accord avec la méthode de production elle-même : les idées approuvées par la direction de la chaîne sont réalisées sur place, dans des décors locaux et par les professionnels locaux. 


\section{Les productions originales des chaînes de télévision hongroises}

C'est précisément ainsi que fonctionne le marché hongrois et, plus largement, le marché est-européen. Dans un entretien enregistré en décembre 2018, c'est-àdire au moment de la diffusion de la troisième saison de la série Aranyélet, Eszter Angyalosy, scénariste de la chaîne, a pu affirmer que l'ambition de la chaîne était « de chercher des histoires qui puissent être comprises, vécues, senties et soient importantes dans une perspective universelle en même temps que pertinentes localement $»^{1}$ (2018). Une idée similaire a été exprimée par l'ancienne directrice de production, Katalin Schulteisz, en 2012 quand elle a dit qu'il était très important de donner un " goût hongrois » aux productions de la chaîne (2012). Certes, les six ans qui séparent les deux déclarations expliquent la différence de nuances : en 2012, parmi les productions de HBO Hongrie se trouvaient des films documentaires et deux adaptations, celles de la série When Shall We Kiss (Társas játék) et En analyse (Terápia), cette dernière, du reste, allait devenir de plus en plus indépendante de la franchise au cours de la deuxième et de la troisième saison. En 2018, la troisième et dernière saison d'Aranyélet a marqué une étape très importante dans l'histoire de HBO Hongrie : les producteurs locaux ont prouvé qu'ils étaient capables de monter une histoire autonome, car, même si Aranyélet est une franchise, l'équipe hongroise a montré ses capacités créatives dès le deuxième épisode de la première saison. Comment cela s'est-il passé ? Comment la production de contenu original est-elle devenue un élément fondamental de la stratégie de HBO en Hongrie ? Tout d'abord, il faut prendre en compte l'expansion de $\mathrm{HBO}$ en Europe.

HBO a fait son apparition relativement tôt sur les marchés est-européens : en 1991, la chaine s'est introduite en Hongrie et tout au long des années 1990 et 2000 elle a conquis l'Europe de l'Est. En 1994, elle s'installait en République Tchèque, en 1997 en Slovaquie, en 1996 en Pologne, en 1997 en Roumanie, en 2002 en Croatie et en Slovénie et en 2004 en Serbie, au Monténégro et en Bosnie-Herzégovine. En raison du faible nombre d'acteurs sur les marchés télévisuels de ces pays, HBO eut la possibilité de lier son nom à la création de contenus de qualité tout en offrant une possibilité importante aux jeunes professionnels désireux de se manifester sur la scène internationale. Travailler pour HBO était considéré un marqueur de prestige. Un autre fil de l'histoire de HBO en Europe, au sens large, est celui de la collaboration sur les marchés ouest-européens où $\mathrm{HBO}$ était déjà présent grâce au réseau Sky Atlantic (Autriche, Allemagne, Irlande, Italie, Suisse, Grande-Bretagne), en France à partir de 2008 à travers OCS City Generation HBO, aux Pays-Bas à partir de 2012 grâce à la société Ziggo. De notre point de vue, la naissance de HBO Nordic, en 2012, est également importante. C'est alors qu'en collaboration avec Parsifal International, HBO est devenue en même temps accessible en Finlande, en Suède, en Norvège et au Danemark. Les territoires les 
plus récemment conquis par l'empire HBO sont l'Espagne, en 2016, et le Portugal, en 2019. La création de HBO Nordic est particulièrement significative, car elle témoigne le plus clairement d'une stratégie procédant par unités régionales dont les contenus puissent être diffusés selon une seule et même logique géographique, au sein de laquelle les différences entre les unités culturelles, pays ou microrégions, s'effacent. Mon propos dans cet article n'est pas de formuler une critique de cette stratégie, je constate simplement son existence. Dans ce sens, les contenus d'Europe de l'Est entrent dans une même catégorie de production locale. Les productions fabriquées en Europe de l'Est se caractérisent en effet par le même processus : les scénarios sont développés dans le contexte local par des professionnels locaux, mais leur qualité doit être approuvée dans le centre londonien de HBO Europe. Cette manière de faire, qui explique le recours au système de la franchise, garantit la cohérence des contenus de la chaîne.

Anikó Imre attire notre attention sur le fait que l'histoire de HBO Europe doit être insérée dans le cadre de l'évolution locale des marché télévisuels : son intention est de prouver que HBO ne tient pas véritablement compte des traditions locales et, au contraire, contribue à mettre en évidence, au sein même des unités culturelles, la différence entre, d'une part, le public orienté vers les formats classiques s'appuyant sur des traditions locales et, d'autre part, le public plus averti, consommateur de films d'auteur - une différence qui est déjà très visible dans le cas des audiences (Imre, 2018, 61-63). Plus concrètement - ou encore dans la perspective de la production - de cette analyse on peut déduire que la collaboration avec des professionnels locaux n'engage pas l'intégration véritable d'un savoir-faire et d'une tradition locale. De notre point de vue, il est intéressant de constater que la stratégie de $\mathrm{HBO}$, aux débuts de la production originale de contenu en Hongrie, consista à rester conforme aux attentes du public hongrois en optant pour l'adaptation de la franchise When Will We Kiss, jugée par les producteurs comme étant assez proche de la tradition hongroise des comédies du grand écran. ${ }^{2}$ Le format des séries dramatiques a été adapté pour la première fois avec Terápia, une série dont l'adaptation pouvait être considérée comme une entreprise sans risque, en raison des facilités techniques de réalisation (la trame se développe dans un espace clos et l'évolution des personnages domine l'intégralité de la production). Par ailleurs, cette production est devenue de plus en plus audacieuse dans l'intégration des réalités locales à partir de la deuxième et plus encore à partir de la troisième saison. En ce qui concerne les séries criminelles et les thrillers, format de prédilection de la chaîne HBO, elles n'ont été adaptées que dans une troisième phase, quand la chaîne a jugé le public hongrois et les producteurs locaux prêts à la réception du format. ${ }^{3}$ Or, le succès d'Aranyélet sur le marché local a été tellement important qu'une autre chaîne de télévision commerciale hongroise, RTL Klub, a jugé opportun d'investir dans une série criminelle originale, Alvilág, qui a commencé à être diffusé récemment. 


\section{La mise en scène et son rôle dans les productions télévisuelles}

Dans le format des séries télévisées que nous venons de présenter brièvement, nous pouvons remarquer un intérêt pour le local, du point de vue géographique et culturel et, parallèlement, une attirance pour l'authentique. C'est-à-dire que l'on s'efforce de mettre en scène des décors qui soient conformes avec les réalités locales et capables d'illustrer avec vraisemblance l'origine et l'évolution des personnages. D'une part, toute une évolution du décor filmique peut être évoquée, quand on pense à la transformation des villes et autres paysages en espaces de tournage, processus qui a encouragé la logique du tourisme culturel (Gravari-Barbas, 1999). Ces changements ont eu un impact important, à commencer par la naissance du regard consommateur sur les espaces, décrit par John Urry (1995, 1-15) ou par tout ce qui entre aujourd'hui dans le domaine du marketing territorial.

D'autre part, nous assistons à l'évolution et à l'émancipation du décor de film et des professions associées. Parallèlement à cette évolution, on peut observer l'émergence d'un discours scientifique appliqué au décor de film (Barsacq 1985, 10-11). Tout d'abord, on remarque un intérêt pour la nature éphémère et fragmentée du décor filmique, pour son "invisibilité" qui part du constat que l'on doit créer l'illusion d'un espace intégral, mais qui n'existe pas dans la réalité (Bergfelder-Harris-Street 2007, 13-14). À partir des années 1990, les directeurs artistiques et les chefs décorateurs de films ont attiré l'attention sur l'importance de leur travail et leurs efforts commencent à être reconnus.

C'est aussi le moment où deux types de discours scientifique sur l'analyse du décor ont fait leur apparition : le premier est centré sur le rapport entre le décor et la narration, le deuxième sur l'effet émotionnel produit par le décor sur son audience. Les premiers critères d'analyse sont apparus dans le livre de Charles et Mirella Jona Affron, qui envisagent cinq catégories de décors : (1) dénotation (denotation) - l'effet de réel produit par le choix d'un emplacement de tournage ; (2) ponctuation (punctuation) - le rôle des emplacements pour souligner les points culminants de la narration ; (3) embellissement (embellishement) - le rôle rhétorique des emplacements attirant l'attention du spectateur sur le contexte spatial et démontrant, au moyen du décor, les nécessités narratives de l'histoire ; (4) artifice (artifice) - le rôle métaphorique du décor volontairement construit dans le but d'attirer l'attention sur sa propre nature artificielle et illusoire - voir par exemple les décors de Casanova de Fellini (1976) où le design évoque une certaine période historique dont l'existence en tant que représentation mentale est non conforme à la réalité historique ; (5) intensité du design (design intensity) pour désigner le cas particulier de l'emploi du décor dans un film où un espace clos encapsule toute la narration et inspire en tant que tel l'évolution de l'intrigue dans son intégralité. Pour cette catégorie, nous pouvons citer La Corde (1948) 
d'Alfred Hitchcock, où toute l'histoire se déroule dans le même appartement et la tension dramatique s'accroît justement à cause de l'impossibilité de sortir du lieu en question (Affron, 1995, 36-60).

En 1998, Charles Tashiro a introduit une nouvelle taxinomie dans l'analyse du décor de film en dépassant l'idée que celui-ci est un simple accessoire de la narration. Tashiro affirme en effet que le décor peut être interprété et classé selon l'effet qu'il produit sur le spectateur et selon son interaction avec l'évolution et la caractérisation des personnages du film. C'est-à-dire que le décor fonctionne comme l'espace qui nous entoure et qu'il s'organise autour du personnage comme une succession de cercles concentriques : les objets qui se trouvent sur le corps du personnage (vêtements, bijoux, maquillage) puis les réalités matérielles de l'espace immédiat (meubles et objets), puis l'espace habitable (maison, bâtiments), l'espace qui peut être parcouru (les rues, les boulevards) et les paysages (espaces plus lointains, villes, etc.), et finalement l'espace cosmique. De ce point de vue, une dimension importante dans l'usage du décor est le niveau auquel le réalisateur place la caméra. Tashiro attire l'attention sur le fait que différentes approches dans la mise en scène correspondent à différents usages du décor : c'est-à-dire que le design peut jouer un rôle plus ou moins symbolique selon les intentions du réalisateur. Un autre aspect important est celui des relations entre le temps et le décor : si le décor contient des objets que l'on peut facilement dater, il deviendra historique, donc la temporalité jouera un rôle important ; par contre, des films comme ceux de Béla Tarr construisent des espaces atemporels en s'appuyant sur des objets sans âge (Tashiro 6-56).

Cette approche peut être confrontée à la conception de Bergfelder, Harris et Street, selon laquelle les films, par leur décor, construisent un espace mental, un espace haptique dans lequel nous avons nos points de repère et où l'on s'oriente à l'instar des espaces physiques : l'espace filmique a une architecture à soi (Bergfelder, Harris et Street 2007, 21-25).

Toutes ces caractéristiques du décor cinématographique doivent être reconsidérées dans le contexte des arts de la télévision : même si de nombreux parallélismes existent entre le travail d'un directeur artistique de films et celui des séries télévisées, le travail pour le petit écran présuppose la construction d'un univers valable pour une période plus longue, capable d'assurer le cadre de vie de personnages dans une conception sérielle (Cotté 1962, Delavaud 2005). C'està-dire qu'il existe un rapport dynamique entre les décors sériels et les décors épisodiques : les décors sériels assurent la cohérence de l'univers d'une série et mettent les épisodes en relation les uns avec les autres, quant aux décors épisodiques, ils sont en relation avec les événements et peuvent considérablement varier au gré des évolutions du scénario (Hansen - Waade, 2017, 129).

Finalement, notre dernière considération, en ce qui concerne l'analyse du décor des séries télévisées, est l'idée du décor compris comme valeur de production : 
cette approche permet de relier la première et la deuxième partie de cette courte présentation du rôle du décor dans la production, car elle met l'accent sur le fait que la valorisation des espaces physiques dans l'esthétique télévisuelle contribue à la communication de l'image de ces lieux tout en établissant, en même temps, un message symbolique construit selon les nécessités de la narration. ${ }^{4}$ Dans l'analyse qui suit nous allons essayer de rendre compte du rôle joué par le décor dans les trois univers sériels évoqués au début de notre article.

\section{Le décor dans Terápia, Aranyélet et Alvilág}

Dans le choix des séries télévisées, nous nous sommes appuyés sur le critère de la production originale et sur celui du genre cinématographique. Nous avons opté pour des séries dramatiques et criminelles parce qu'il nous a semblé que l'intérêt pour la construction réaliste des décors y est plus grand que dans le cas d'autres genres. C'est pourquoi trois séries hongroises en particulier se sont imposées comme dignes d'être analysées : la version hongroise de la série En analyse, Terápia (trois saisons, 2012-2017), la série Aranyélet (trois saisons, 2015-2018) et finalement une série récente, Alvilág, qui vient de débuter sur la chaîne RTL Klub, dont seulement les deux premiers épisodes ont été diffusés au moment de l'écriture de cet article.

En ce qui concerne les deux premières séries, le fait qu'elles se soient toutes les deux arrêtées après la troisième saison n'est pas un hasard : les créateurs considèrent généralement qu'un cycle de trois saisons permet de tout donner en matière de savoir-faire filmique et à partir de la quatrième saison, s'il est bien évidemment possible d'innover sur le plan narratif, les effets filmiques risquent d'être, quant à eux, répétitifs (Angyalosy et al 2018). Nous savons que les décors de ces deux séries produites par la chaine $\mathrm{HBO}$ ont été très minutieusement sélectionnés. Leurs créateurs se sont concentrés sur le sens symbolique donné aux espaces. Nous allons voir d'ailleurs que la fin de la série Aranyélet offre la possibilité d'une interprétation du destin des personnages qui est directement liée à un type déterminé d'espace, l'immeuble en béton de type socialiste, le panel, un type d'habitation méprisé par la principale protagoniste de la série, Janka. Chacune des trois séries dessine la géographie symbolique de la même ville, Budapest, une ville largement utilisée dans l'industrie filmique en tant que décor simulant d'autre villes où il est bien plus cher de tourner des films. Au-delà de Budapest, les villes provinciales hongroises ne figurent pas dans les séries, cela témoigne justement d'un pays très centralisé. ${ }^{5}$ Il faut tout de même attirer l'attention sur l'ambition de la série Aranyélet de montrer quelques réalités extra-budapestoises : on observe en particulier la présence de plusieurs villages. Du reste, il s'agit, très symboliquement, de lieux où les nouveaux-riches sont en 
mesure de dissimuler leur fortune sans craindre d'être interrogés par les autorités locales.

La direction artistique de la première saison de Terápia a été assurée par Csaba Stork, décorateur de cinéma connu pour avoir contribué à des long métrages comme Hellboy 2 et Underworld. HBO a donc recruté un décorateur doté d'une riche expérience internationale. C'est lui qui a personnellement conçu le décor où se déroulent les séances d'analyse de la série. Les chroniqueurs d'une visite organisée sur les lieux du tournage ont documenté la manière dont les lieux de travail des deux thérapeutes (le protagoniste et son superviseur) furent construits, soulignant le fait que les acteurs eux-mêmes ont pu donner leur avis sur la sélection du mobilier et des objets (Simor-Gaines 2012). La logique de la production se fonde sur le concept de l'intensité du design, étant donné que les relations entre les personnages, leur évolution et la trame de la narration sont largement gouvernés par l'espace et les espaces de l'analyse. Les choix géographiques se sont portés sur le centre de la ville, la maison du psychologue à Buda (rue Garas) et l'appartement du superviseur, une psychologue également, à Pest (rue Szalay) - les deux décors ont été bâtis en studio. Un changement radical dans le récit a lieu au début de la deuxième saison, quand le psychologue déménage de sa maison familiale et commence une vie de célibataire : son nouvel appartement se trouve à Pest, dans le quartier fréquenté par les jeunes. D'ailleurs, c'est dans ce quartier que se trouve également le cabinet de sa nouvelle psychologue-superviseur, qui a été conçu dans le but d'évoquer un contraste avec les deux autres cabinets : c'est un espace lumineux et décoré selon les principes du design scandinave. Les critiques ont remarqué que les décors utilisés dans la série témoignent d'une aisance matérielle assez peu vraisemblable, si on la compare au niveau de vie de la population hongroise : Ildikó Enyedi, une des directrices de la saison, a répliqué qu'ils s'étaient, au contraire, efforcés d'être attentifs aux réalités sociales et avaient choisi les lieux et le décor en tenant compte des possibilités financières des héros. En l'espèce, il faut prendre en considération le statut social des personnages de la série : ils sont tous membres des catégories aisées de la population hongroise, seules, d'ailleurs, ayant les moyens de s'offrir des séances d'analyse et capables de reconnaître l'utilité d'une telle dépense. En ce qui concerne le psychologue luimême, héros de la série, qui déménage seul dans un appartement spacieux pour $\mathrm{y}$ vivre et y exercer sa profession, il faut remarquer qu'il est le seul personnage d'une série hongroise (et même d'une séries est-européenne produite par HBO) décidant de vivre seul et pouvant se le permettre : vivre seul en Europe de l'Est est une option possible pour une couche très mince de la société est-européenne. La série se réfère d'ailleurs à l'image touristique de Budapest : plusieurs plans d'ensemble montrent l'image d'une ville qui se donne au regard touristique, dont les rues sont pleines de touristes et dont les différents lieux semblent tous destinés aux visiteurs étrangers. Le dernier épisode de la série se termine par une scène 
dans laquelle le protagoniste regagne la ville en s'échappant des cabinets clos liés à son identité professionnelle.

En plus de Budapest et ses espaces clos ou destinés aux touristes, la série Aranyélet, quant à elle, montre plusieurs lieux situés dans diverses quartiers du tissu urbain. Étant donné la nature éminemment narrative de la série, nous allons utiliser les catégories des Affron pour l'analyse des décors successifs et nous allons finalement démontrer comment le choix des lieux de tournage illustre l'évolution psychique de la protagoniste, Janka Miklósi, jouée par Eszter Ónodi.

Au coeur de la série se trouvent les événements de la vie d'une famille, les Miklósi, qui au début se situe encore aux marges des bas-fonds de Budapest et réussissent peu à peu à se hisser jusqu'au sommet de la maffia hongroise. L'évolution de leur statut est symbolisée par les changements successifs de lieu d'habitation : on assiste à pas moins de cinq déménagements. La première maison est une vaste villa construite en style moderniste, à Budaliget : le luxe apparaît notamment dans la présence du sauna, mais sans bassin, selon les tendances de 2014. L'aspect le plus mémorable de cette maison est le papier peint de la salle à manger avec ses grands motifs graphiques alternant le gris clair et foncé. Le décor choque par l'absence d'objets personnels, la pauvreté du détail : nulle collection d'objets (livres, petits souvenirs, DVDs, etc.) caractéristique des maisons plus accueillantes. Le téléspectateur a l'impression que la famille réside ici depuis peu et que ses membres n'ont pas eu le temps de s'approprier leur lieu de vie. On sait également qu'ils sont mal vus par leurs voisins, surtout un vieux couple aisé qui exprime sans détour l'avis que les Miklósi sont de parvenus peu dignes de l'amitié des habitants du voisinage. Le style de la maison a été choisi pour caractériser l'état d'esprit de la mère de famille. Comme le décorateur, Balázs Hujber, l'affirme : «Il était important que tout reflète le goût de Janka ou, plus exactement, son manque de goût, le fait que la protagoniste n'a pas de style propre, elle s'inspire des magazines de décoration et si elle voit quelque chose en première page, elle l'achète sur le champ. Janka ne voit pas la cohérence des choses, mais seulement des éléments épars. Il a fallu concevoir son entourage en nous inspirant de cela. » (2015) L'intention du décorateur était de réaliser un espace impersonnel, qui s'accorde avec la personnalité de la protagoniste, toujours à la recherche des symboles du statut social sans avoir elle-même le sens du style. Quand, au cours de la deuxième saison, la famille commence une nouvelle vie dans l'ancienne maison familiale du père de famille, on ne perçoit chez elle aucun regret pour la maison perdue : Janka regrette la richesse perdue, mais pas le lien spirituel qui aurait pu exister entre elle et sa maison.

Le deuxième espace des Miklósi est la maison familiale d'Attila, le père. Ils y déménagent afin de recommencer une vie honnête, dont seront exclues toutes les tricheries anciennes. La décision, prise par le père, est vivement contestée par les autres membres de la famille. Le nouveau logis familial reflète la vie aisée des 
années 80 : le mobilier semble vieilli, il y a beaucoup de souvenirs et les objets du décor contribuent à donner l'impression d'une famille ayant une histoire dont elle est fière. En opposition avec la maison de la première saison, la lumière manque dans cet espace et les détails, assurant la sensation de familiarité, provoquent également un effet suffoquant. C'est exactement l'impression que ressent Janka a dans cette intérieur-refuge : elle essaie de se conformer à cette nouvelle vie, de faire régulièrement le ménage, mais tous ses gestes sont brusques et maladroits, elle donne l'impression de vouloir s'évader à tout moment. C'est ce qu'elle finit par réaliser elle-même. Elle quitte son domicile et s'installe chez Endre Hollós, ancien ami de la famille, parrain de son fils et criminel puissant dont les relations s'étendent jusqu' aux milieux politiques, noyau d'un cercle de la maffia hongroise.

La troisième maison est donc la villa Hollós, dont le style impersonnel évoque celui de la première saison, mais les symboles de la richesse y sont bien plus forts : grands espaces vides, meubles en verre, bassin de natation, statues. Cette maison évoque le style américain et reflète le rêve américain auquel un gangster comme Hollós s'adonne entièrement. Il est donc naturel que, dans cette maison, on ne boive que du whiskey, on joue souvent aux cartes et que tous les éléments du décor reflètent le style de vie d'un riche célibataire. La première chose à faire, pour Janka, c'est de "personnaliser" cet espace, une tâche dont elle s'acquitte de la même manière que dans sa maison précédente : elle achète des tapis dernier cri, se délecte du rôle de celle qui s'y connaît en matière de décoration intérieure.

La maison la plus spectaculaire est sans aucun doute la villa de la dernière saison, dont le large panorama s'ouvre sur le coude du Danube. Il est notoire que sa vente, après le tournage, a donné lieu à de nombreux commentaires dans la presse hongroise. Le choix de cet emplacement fut un geste médité avec soin par les réalisateurs : leur intention était de témoigner d'un saut majeur sur l'échelle sociale. Une manière d'illustrer ce saut était un déménagement hors de Budapest. La villa, avec son panorama, devait donner l'idée que cette famille était arrivée à dominer le paysage hongrois. Le lac Balaton et ses célèbres villas aurait pu s'imposer comme choix, mais les réalisateurs voulaient montrer que les nouveaux riches sont à la recherche de lieux plus singuliers, leur dessein étant la création de nouveaux centres de pouvoir. Le coude du Danube était, en ce sens, un choix stratégique. C'est à Dunakeszi que l'on a finalement déniché la villa recherchée. Dans la troisième saison, un autre personnage, Feri Gáll, joue un rôle crucial, il est plus puissant encore que Hollós, c'est un homme d'affaires qui joue en grand. C'est lui le premier à quitter la capitale, pour s'installer dans un ranch qui se situe dans les environs de Zirc. En réalité, il se cache pour être en mesure d'exercer son pouvoir sans attirer l'attention des médias.

La villa de la troisième saison est le premier endroit où Janka se sent à son aise et pense avoir enfin atteint le but qu'elle a toujours poursuivi. Cette villa joue un rôle éminemment symbolique dans la série : dans le septième épisode, 
d'ailleurs très mémorable, les créateurs se risquent dans un exercice de style courageux et optent pour la technique de l'intensité du design, technique évoquée plus haut à propos de La Corde de Hitchcock. Dans cet épisode, Janka est sur le point d'accoucher, les membres de la famille, son mari, les deux enfants (sa fille et son fils) et le frère de son mari sont tous réunis dans la villa qui est bloquée à cause de la neige. Le suspense est au maximum dans cet épisode, moment où les cinq personnages reviennent sur leur passé tandis qu'ils doivent mettre au monde un enfant dont l'identité condensera justement tout le passé. Enfant de Janka et Hollós, le nouveau-né tue presque sa mère et oblige les membres de la famille de recommencer une nouvelle vie.

Et l'on arrive ainsi à une cinquième habitation familiale : un appartement situé dans un quartier d'immeubles de type socialiste où l'on voit, lors du dernier épisode, la famille au beau milieu des préparatifs de Noël. Les créateurs ont opté pour l'embellissement du design : d'un côté, on se souvient des propos de Janka quand elle disait qu'elle ne retournerait jamais dans l'appartement où elle a commencé sa vie. D'un autre côté, ce quartier est également un symbole médiatique : la série culte de la télévision hongroise, Szomszédok, diffusée entre 1987 et 1999, véritable emblème télévisuel de la transition hongroise, se déroulait dans un quartier de Budapest semblable, qui représentait la vie difficile, mais paisible, des petites gens de Budapest, honnêtes et pleins d'espoir à l'égard du monde nouveau. En faisant ce choix, la série formule un message politique important : pour en finir avec toutes ces histoires de corruption et de criminalité, il faut retourner au moment du changement du régime, il faut effacer les péchés des deux dernières décennies et retrouver la naïveté et surtout la conscience morale des gens des années 1990.

Nous avons donc vu comment le sens dénotatif des emplacements de la série a été calculé selon la logique des évènements, et quelle était la logique des déménagements successifs. En ce qui concerne le procédé de ponctuation, il faut justement s'arrêter sur un exemple, attirant l'attention sur la maison d'enfance de Janka que l'on visite pendant la deuxième saison : on apprend alors qu'elle a vécu dans l'extrême misère, qu'elle a fait dans sa jeunesse l'expérience d'un père abusif et qu'elle s'est évadée de cette situation invivable avec l'ambition de construire une vie complètement différente de celle qui lui était destinée. Cette maison fonction comme une ponctuation au sein de l'évolution psychologique du personnage.

En définitive, la série Aranyélet propose une cartographie complète de la société hongroise postsocialiste, en mettant l'accent sur les lieux de tournage choisis pour illustrer quelques tendances et styles de vie tout en construisant une sorte d'utopie spatiale. La fin de la troisième saison transforme cette fiction en une série à clef qui explique et dénoue, dans un certain sens, les conflits majeurs de la société hongroise contemporaine. 
Quant à la série Alvilág, après seulement deux épisodes diffusés, nous pouvons constater qu'une attention évidente est accordée aux décors, mais, d'un point de vue sociologique, elle est moins avertie qu'Aranyélet. La nouveauté de cette série réside dans l'utilisation des images panoramiques, des plans de grand ensemble devenus très caractéristiques du style du réalisateur, Károly Mészáros Ujj. L’influence du "Nordic noir" est indéniable : les couleurs, les ombres, les paysages, les prises de vue des rues de Budapest pendant la nuit sont tous conçus selon cette esthétique.

Les trois cas présentés ici avec plus ou moins de minutie démontrent la manière dont la direction artistique des séries hongroises essaie de traduire les changement sociaux et économiques du pays. Une recherche certaine de la qualité est un élément digne d'être remarqué. Du point de vue de la représentation de la culture matérielle, la conclusion suivante s'impose : la culture matérielle des Hongrois, et surtout celle des nouveaux riches qui se considèrent gagnants du changement de régime, est caractérisée par un manque d'intérêt pour le détail, pour le vécu et pour l'authentique. À quel moment ces gens ont-ils cessé de s'identifier à leurs objets ? La réponse à cette question est la même que celle donnée à la question centrale posée par Aranyélet : quand la société hongroise a-t-elle échoué dans son projet de construire une démocratie vivable pour tout le monde ?

\section{Références}

Affron, Charles - Affron, Mirella Jona, 1995. Sets in Motion: Art Direction in Film and Narrative. New Brunswick : Rutgers University Press.

Angyalosy, Eszter - Mátyássy, Áron - Végh, Zsolt, 2018. Élő beszélgetés az Aranyélet alkotóival. Zoom, [Online]. 04/12/2018, Available at : https://www.facebook.com/zoomponthu/ videos/603834916721803/?tn-str=k*F [Accessed 12. 02. 2019].

Barsacq, Léon, 1985. Le Décor de film. 1895-1969. Paris : Henri Veyrier.

Bergfelder, Tim - Harris, Sue - Street, Sarah, 2007. Film Architecture and the Transnational Imagination: Set Design in 1930s European Cinema. Amsterdam : Amsterdam University Press.

Cotté, Jean, 1962. Le décor de télévision. Études cinématographiques 16-17 (2).

Delavaud, Gilles, 2005. L'art de la télévision. Histoire et esthétique de la dramatique télévisée (1950-1965). Paris : De Boeck Supérieur.

Dezső, András, 2018. Alvilág az Aranyéletben. Fikció vs. valóság. Index, [Online]. 10/10/2018, Available at : https://index.hu/kultur/2018/10/10/alvilag_az_aranyeletben_fikcio_vs._valosag/ [Accessed 12. 02. 2019].

Gelencsér, Gábor, 2017. The Paradox of Popularity. The Case of Socialist Crime Movie in Hungary. In: Ostrowska, Dorota - Pitassio, Francesco - Varga, Zsuzsanna, ed. Popular Cinemas in East Central Europe. Film Cultures and Histories. London: I. B. Tauris, 85-102.

Gravari-Barbas, Maria, 1999. La ville décor : accueil de tournages de films et mise en place d'une nouvelle esthétique urbaine. Cybergeo : European Journal of Geography [Online], document 101, 27/05/1999, Available at : http://journals.openedition.org/cybergeo/1170 [Accessed 12. 02. 2019]. 
Hansen, Kim Toft - Waade, Anne Marit, 2017, Locating Nordic Noir. From Beck to The Bridge, London : Palgrave Macmillan.

Hauschel, Tamás, 2015. Ott voltam az Aranyélet 3. forgatásán, de nem beszélhetek róla. Player [Online]. 2018/10/11, Available at https://player.hu/kult/ott-voltam-az-aranyelet-3-forgatasan-de-nem-beszelhetek-rola/ [Accessed 12. 02. 2019].

Hujber, Balázs, 2015 : Miről árulkodik a Miklósi-villa? HBO. [Online]. 2015/12/01, Available at https://www.hbo.hu/series/aranyelet/encyclopedia/mirol-arulkodik-a-miklosi-villa_52329 [Accessed 17. 02. 2019].

Imre, Anikó, 2018. HBO's e-EUtopia. Media Industries, 5.2, 50-68.

Mittel, Jason, 2015. Complex TV: The Poetics of Contemporary Television Storytelling. New York : New Work University Press.

Pinsolle, Dominique - Rindel, Arnaud, 2017. Comment HBO a inventé la série de qualité? Le Monde diplomatique, Manières de voir, 154, 32-34.

Schulteisz, Katalin, 2012. Legyen valami magyar íz. Vodál Vera interjúja Schulteisz Katalinnal, az HBO executive producerével. Filmtett, [Online], 26/03/2012, Available at http://www.filmtett. ro/cikk/3019/interju-schulteisz-katalinnal-az-hbo-executive-producerevel [Accessed 17. 02. 2019].

Simor - Gaines, 2012. Így készült a Terápia (forgatási napló). Sorozatjunkie, [Online], 2012/10/12, Available at http://www.sorozatjunkie.hu/2012/10/12/igy-keszult-a-terapia-forgatasi-naplo-1resz---irta-symor-es-gaines/ [Accessed 17. 02. 2019].

Soós, Tamás, 2018. Beszélgetés Krigler Gáborral és Tasnádi Istvánnal az Aranyéletről. Revizoronline, [Online] 11/12/2018, Available at https://revizoronline.com/hu/cikk/7649/beszelgetes-krigler-gaborral-es-tasnadi-istvannal-az-aranyeletrol/_[Accessed 17. 02. 2019].

Tashiro, Charles S., 1998. Pretty Pictures : Production Design and the History of Film. Austin : University of Texas Press.

Urry, John, 1995. Consuming Places. London and New York : Routledge, Taylor and Francis.

Varga, Balázs, 2017. The Missing Middle: Transformations and Trends in Hungarian Film Comedies after Political Change. In: Dudková, Jana - Misiková, Katarína, ed. Transformation Processes in Post-Socialist Screen Media. Institute of Theater and Film Research, 97-117.

Wolff, Michael, 2015. Television is the New Television. The Unexpected Triumph of Old Media in the Digital Age. New York : Penguin.

\section{Filmographie}

Szomszédok, 1987-1999, réalisé par Ádám Horváth, produite par MAHIR, Magyar Televízió Művelődési Főszerkesztőség

Aranyélet, 2015-2018, réalisé par Áron Mátyássy, Zsombor Dyga, produite par HBO Europe

Alvilág, 2019, réalisé par Károly Mészáros Ujj, produite par RTL Klub

Terápia, 2012-2017, réalisé par Attila Gigor, Ildikó Enyedi, produite par HBO Europe

Társas játék, 2011-2013, réalisé par Gábor Herendi, produite par HBO Europe 


\section{Notes}

1 „Olyan történeteket keresünk, amik univerzálisan érthetőek, érezhetőek, átélhetőek és fontosak, ugyanakkor lokálisan relevánsak."

2 Pour l'évolution des comédies après le changement de régime voir Varga, 2017.

3 Sur l'histoire tourmentée des narrations policières en Hongrie voir l'étude de Gelencsér, 2017.

4 Ce type d'analyse de l'utilisation des éléments du décor dans les récits de 'Nordic noir' scandinave peut être lu dans le livre de Hansen-Waade, 2017: 77-81.

5 Gravari-Barbas met l'accent une situation similaire dans le cas des villes françaises à la fin des années 1990 : contre la prédominance de Paris dans l'industrie filmique, les villes de province étaient contraintes de construire leur propre image de marque pour se faire remarquer par les réalisateurs.

Open Access. This is an open-access article distributed under the terms of the Creative Commons Attribution 4.0 International License (https://creativecommons.org/licenses/by/4.0), which permits unrestricted use, distribution, and reproduction in any medium, provided the original author and source are credited, a link to the CC License is provided, and changes - if any - are indicated. (SID_1) 